\title{
Modelización aplicada al diseño de sistemas de control en el horno alto ${ }^{\left({ }^{*}\right)}$
}

\author{
R. Rosal ${ }^{(*)}$, C. Blanco ${ }^{(*)}$, M. Díaz ${ }^{(*)}$ y J. Sáiz $\left.{ }^{(* *}\right)$ \\ Resumen El control del proceso de fabricación de arrabio en hornos altos resulta complejo debido a las \\ condiciones de operación: conocimiento incompleto de la quimicofísica de los procesos que tienen \\ lugar en el interior del horno, grandes dimensiones del reactor que se traducen en tiempos muertos \\ considerables y constantes de tiempo elevadas que provocan una gran inercia a las acciones de \\ control. En este trabajo, se ha planteado un modelo matemático por zonas que permite describir el \\ comportamiento del horno excepto el crisol, se han identificado sus parámetros y se ha obtenido el \\ perfil interno de temperaturas y composiciones. El análisis del modelo permite predecir los efectos \\ de un cambio en cualquier variable del sistema así como desarrollar un algoritmo de control \\ automático.
}

Palabras clave: Horno alto. Control de procesos. Modelo matemático.

\section{Modelling applied to the design of blast furnace control systems}

\begin{abstract}
The production of pig iron in blast furnaces resists automatic control strategies due to the lack of knowledge about physical and chemical phenomena taking place inside the reactor. High dimensions lead to important dead times and lags. As a consequence it is very difficult to quantify control actions from actual process measurements. A simplified multizonal mathematical model has been proposed that allowed the description of a given blast furnace excluding hearth. Parameters underlying the model have been identified and, under appropriate assumptions, temperature and composition profiles have been established. The analysis of model predictions has been illustrated with steadystate responses to typical control actions.
\end{abstract}

Keywords: Blast furnace. Process control. Mathematical model.

\section{INTRODUCCIÓN}

El horno alto es un reactor gas-sólido-líquido donde tiene lugar la formación de arrabio a partir de mineral de hierro, coque, fundentes y aire. El tratamiento de dicho reactor resulta complejo tanto por sus dimensiones físicas como por la diversidad de reacciones que en él tienen lugar. Actualmente, el horno alto se regula casi exclusivamente por las acciones del operador. El importante retardo que se produce desde la alteración de las variables de pro-

(•) Trabajo recibido el día 27 de junio de 1994.

${ }^{*}$ Dpto. de Ingeniería Química. Universidad de Oviedo. Julián Clavería, s/n. 33071-Oviedo (España).

(**) División de Informática de Procesos y Automatización Industrial. ENSIDESA. 33400-Avilés (España). ceso hasta la determinación de sus consecuencias en las corrientes de salida, unido a la complejidad de las reacciones químicas que tienen lugar dentro del horno, son factores que provocan que las acciones de control del operador sean muy difíciles y con frecuencia conduzcan a una inestabilidad adicional. Como consecuencia, el horno alto presenta una acusada tendencia a funcionar de forma errática y lejos de una situación óptima desde el punto de vista económico. Las estrategias de control habituales sólo permiten una predicción semiempírica de las calidades finales y una determinación no cuantitativa de la influencia de los distintos parámetros de operación sobre las características finales del arrabio que se desea controlar.

Para el desarrollo de modelos matemáticos, es necesario conocer las reacciones que tienen lugar en las diferentes zonas del horno alto. Sin embargo, 
ictualmente existen pocos datos que informen sobre las condiciones internas del mismo y la evoución de las zonas de reacción en función de las zondiciones de operación.

En este trabajo se describe un modelo matemático que se ha desarrollado con el objetivo de permitir el establecimiento de un sistema de control flexible, a fin de limitar al mínimo las oscilaciones de calidad del producto y emplear al máximo las materias primas siderúrgicas. El modelo considera todo el horno con excepción del crisol y constituye la base de un trabajo más amplio cuyo objetivo es desarrollar un método de seguimiento y control del horno alto. Se ha decidido afrontar el problema de la modelización empleando un modelo multizonal no estacionario en el que los balances de materia y de energía se han resuelto simultáneamente para todas las zonas. Las temperaturas, composiciones y propiedades de los materiales pueden variar de una zona a otra y en función del tiempo de operación.

\section{MODELO MATEMÁTICO}

La obtención de arrabio en el horno alto se realiza a partir de mineral de hierro, coque, fundentes, aire y vapor de agua como materias primas, obteniéndose arrabio, escoria, gases y polvo de tragante como productos finales. El arrabio debe cumplir ciertas especificaciones de composición motivadas, fundamentalmente, por las limitaciones impuestas en la acería. Debido a la complejidad del horno alto y a la dificultad de desarrollar un modelo matemático que tenga en cuenta de forma precisa la totalidad de los fenómenos fisicoquímicos que tienen lugar en él, se han adoptado las simplificaciones que se mencionan a continuación:

- Se ha supuesto que todas las variables (composiciones, temperaturas o cualquiera otra) son constantes en dirección radial.

- El horno se ha dividido en zonas caracterizadas por una uniformidad de comportamiento (1). El planteamiento se ha completado asignando a cada zona una o varias de las reacciones químicas que tienen lugar en el sistema, así como unas condiciones determinadas de presión y temperatura (2). El crisol no se ha tenido en cuenta en el desarrollo que sigue y quedaría descrito por una o varias zonas dispuestas en serie con las tratadas en este trabajo.

- Se ha supuesto, también, que no se produce acumulación de ningún componente en las zonas que se han considerado. Esto implica que el balance de materia se encuentra en estado estacionario en todo momento, aunque esto no significa necesariamente que las velocidades de reac- ción sean constantes si la alimentación del horno no varía.

- Se han considerado únicamente los componentes principales tanto del sólido como de la corriente gaseosa. En el caso de los componentes reactivos de cada zona, se ha supuesto que todos (excepto el carbono en la reacción de Boudouard) reaccionan en la que les corresponde hasta el consumo total del reactivo limitante de la reacción en la que se encuentra implicado. De acuerdo con lo anterior, el horno se ha dividido axialmente en las cinco zonas siguientes (Fig. 1):

1. Zona superior o de precalentamiento. En esta zona tiene lugar el calentamiento de las cargas y la evaporación del agua que se carga junto con el coque o el mineral.

2. Zona de reducción del óxido de hierro $\mathrm{Fe}_{2} \mathrm{O}_{3}$. En ella se produce la reducción de la hematites a magnetita. Se caracteriza porque la temperatura varía entre 200 y $700{ }^{\circ} \mathrm{C}$ (3).

3. Zona de reserva térmica. En ella la temperatura se mantiene aproximadamente constante a $950{ }^{\circ} \mathrm{C}$. En esta zona se produce la reducción de la magnetita a wustita y la descomposición de los carbonatos.

4. Zona cohesiva. Aquí la temperatura de los sólidos varía entre los 1.050 y $1.500{ }^{\circ} \mathrm{C}$; sin embargo, la mejor forma de determinar esta zona es mediante el análisis de los cambios de gradiente de presión y de temperatura de pared que tienen lugar. En dicha zona se produce la reducción de la wustita tanto por el carbono (directa) como por el monóxido de carbono (indirecta). También se inicia la formación y fusión de escorias y el goteo del hierro líquido (4).

5. Zona de combustión. La temperatura de esta zona es de aproximadamente $2.000{ }^{\circ} \mathrm{C}$. En ella tiene lugar la combustión del carbono del coque, y se encuentra situada a nivel de toberas, limitada en primera aproximación por el "hombre muerto" y la zona cohesiva.

El modelo no considera explícitamente el crisol donde se recogen la escoria y el arrabio, en el que se producen fenómenos de transferencia entre ambos, y cuya temperatura varía entre 1.400 y $1.600{ }^{\circ} \mathrm{C}$ (5). En el caso de ser necesario, su comportamiento podría modelizarse con independencia del resto del horno, en serie con las zonas anteriores.

Cada una de las cinco zonas del modelo se encuentra determinada por una altura de zona llamada $h_{\mathrm{i}}$, a la que corresponde un área de pared lateral del horno, $A_{\mathrm{i}}$, que interviene en el balance de energía para considerar las pérdidas al exterior. Las zonas de reacción intercambian entre sí materia y energía en forma de calor sensible, pero no debido a 


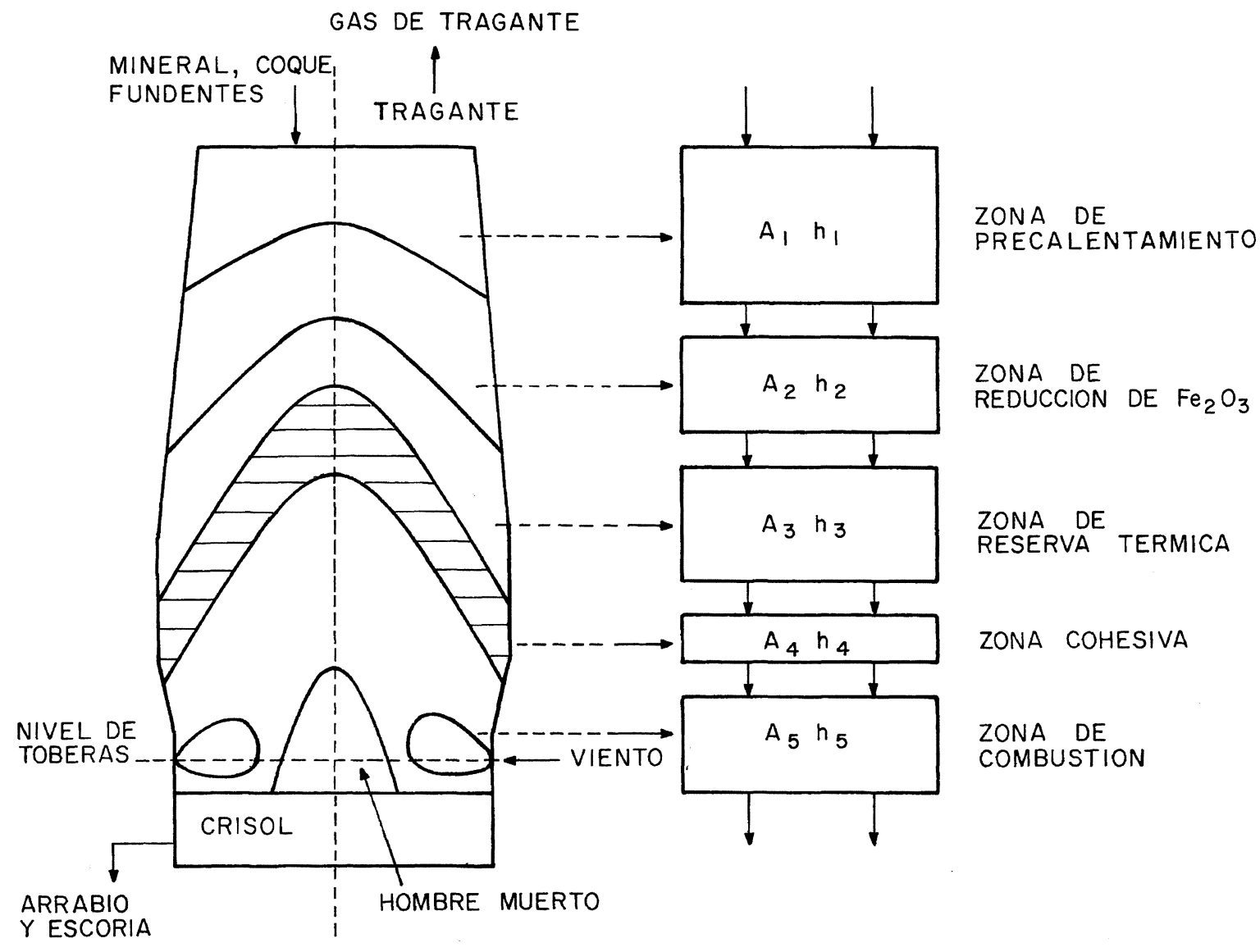

FIG. 1.-Esquema del horno alto y su división por zonas.

Fig. 1.-Blast furnace diagram and division into zones.

mecanismos de conducción y radiación. Dentro de cada zona, se han supuesto constantes las temperaturas del sólido $T_{\text {is }}$ y del gas $T_{\text {ig }}$, así como todas las propiedades físicas que deban considerarse.

Se ha tenido en cuenta un total de diez reacciones que se producen en las distintas zonas del horno. En realidad, cada reacción se encuentra confinada en una sola zona, con excepción de la reacción de Boudouard (reacción del carbono del coque con dióxido de carbono), que tiene lugar a la vez que se produce la reducción de la magnetita $\left(\mathrm{Fe}_{3} \mathrm{O}_{4}\right)$ y de la wustita $(\mathrm{FeO})$; es decir, en las zonas 3 y 4 . Sin embargo, aunque es la misma reacción, se ha tratado como si fuesen dos diferentes, debido a que sus velocidades globales de reacción son distintas.

Las reacciones y transiciones de fase consideradas son las siguientes:

Zona 1. Superior o de precalentamiento. Aunque no hay reacciones químicas, se ha tratado la evaporación del agua incluida en la carga, incluyendo la humedad del coque, como una reacción química cuya velocidad se ha denominado $R_{11}$.

Zona 2. De reducción del óxido de hierro $\mathrm{Fe}_{2} \mathrm{O}_{3}$ : Reacción 1: $3 \mathrm{Fe}_{2} \mathrm{O}_{3}+\mathrm{CO} \rightarrow 2 \mathrm{Fe}_{3} \mathrm{O}_{4}+$ $\mathrm{CO}_{2}$

Zona 3. De reserva térmica:

Reacción 2: $\mathrm{C}+\mathrm{CO}_{2} \rightarrow 2 \mathrm{CO}$ (Reacción de Boudouard).

Reacción 3: $\mathrm{Fe}_{3} \mathrm{O}_{4}+\mathrm{CO} \rightarrow 3 \mathrm{FeO}+$ $\mathrm{H}_{2} \mathrm{O}$.

Reacción 9: $\mathrm{Fe}_{3} \mathrm{O}_{4}+\mathrm{H}_{2} \rightarrow 3 \mathrm{FeO}+\mathrm{H}_{2} \mathrm{O}$. Reacción 10: $\mathrm{CaCO}_{3}\left(\mathrm{MgCO}_{3}\right) \rightarrow \mathrm{CaO}$ $(\mathrm{MgO})+\mathrm{CO}_{2}$.

\section{Zona 4. Cohesiva:}

Reacción 4: $\mathrm{C}+\mathrm{CO}_{2} \rightarrow 2 \mathrm{CO}$ (Reacción de Boudouard).

Reacción 5: $\mathrm{FeO}+\mathrm{CO} \rightarrow \mathrm{Fe}+\mathrm{CO}_{2}$. 
Fusión del óxido de hierro (o del hierro, en función de las entalpías que se utilicen). Dado que sucede en la misma extensión que $\mathrm{R}_{5}$, no es necesario introducir una velocidad adicional, de la misma forma que se hizo con el agua de la carga.

Zona 5. De combustión:

Reacción 6: $2 \mathrm{C}+\mathrm{O}_{2} \rightarrow 2 \mathrm{CO}$

Reacción 7: $\mathrm{C}+\mathrm{H}_{2} \mathrm{O} \rightarrow \mathrm{CO}+\mathrm{H}_{2}$

Reacción 8. $\mathrm{C}_{\mathrm{n}} \mathrm{H}_{\mathrm{m}} \rightarrow \mathrm{nC}+(\mathrm{m} / 2) \mathrm{H}_{2}$

Esta última reacción no se ha tenido en cuenta, puesto que el modelo se ha adaptado a un horno en el que no se inyecta combustible en toberas. Los componentes se han numerado para cada fase de la forma siguiente:

\begin{tabular}{|c|c|}
\hline \multicolumn{2}{|r|}{ Fase gaseosa: } \\
\hline Componentes & Componentes \\
\hline 1: C & $1: N_{2}$ \\
\hline 2: $\mathrm{Fe}_{2} \mathrm{O}_{3}$ & 2: $\mathrm{H}_{2}$ \\
\hline 3: $\mathrm{Fe}_{3} \mathrm{O}_{4}$ & 3: $\mathrm{H}_{2} \mathrm{O}$ \\
\hline 4: $\mathrm{FeO}$ & 4: $\mathrm{O}_{2}$ \\
\hline 5: Fe & 5: CO \\
\hline 6: $\mathrm{CaCO}_{3}+\mathrm{MgCO}_{3}$ & 6: $\mathrm{CO}_{2}$ \\
\hline $\begin{array}{l}\text { 7: } \mathrm{CaO}+\mathrm{MgO}+\mathrm{Al}_{2} \mathrm{O}_{3} \\
\text { 8: } \mathrm{SiO}_{2}\end{array}$ & 7: $\mathrm{C}_{\mathrm{n}} \mathrm{H}_{\mathrm{m}}$ \\
\hline 9: $\mathrm{H}_{2} \mathrm{O}$ & \\
\hline
\end{tabular}

\subsection{Balance de materia}

Se ha planteado un balance de materia separado para las corrientes sólida y gaseosa en cada una de las zonas del horno (6 y 7). Es posible plantear tantos balances de materia como número de componentes multiplicado por el número de zonas. Las variables desconocidas en cada caso son las composiciones de las corrientes de los elementos reaccionantes y las velocidades de reacción. Para la solución del sistema, se ha establecido una relación entre las velocidades $R_{2}$ y $R_{4}$ (reacciones de Boudouard) en las dos zonas en las que tiene lugar por medio de un coeficiente, $\gamma$. Los balances de materia se han formulado como sigue:

Balance de materia global al sólido:

$$
0=\sum_{\mathrm{j}}^{\mathrm{NS}}\left[(s)_{(\mathrm{i}-1) \mathrm{j}}-(s)_{\mathrm{ij}}\right]+\sum_{\mathrm{k}}^{\mathrm{NR}} v_{\mathrm{k}} \delta_{\mathrm{ik}} \mathrm{R}_{\mathrm{k}}
$$

Balance de materia a un componente, $j$, del sólido:

$$
0=(s)_{(\mathrm{i}-1) \mathrm{j}}-(s)_{\mathrm{ij}}+\sum_{\mathrm{k}}^{\mathrm{NR}} \nu_{\mathrm{k}} \delta_{\mathrm{ik}} \mathrm{R}_{\mathrm{k}}
$$

donde $i=1,2,3,4$ y 5 .

Balance de materia global al gas:

$$
0=\sum_{\mathrm{j}}^{\mathrm{NG}}\left[(g)_{(\mathrm{i}+1) \mathrm{j}}-(g)_{\mathrm{ij}}\right]+\sum_{\mathrm{k}}^{\mathrm{NR}} v_{\mathrm{k}} \delta_{\mathrm{ik}} R_{\mathrm{k}}
$$

Balance de materia a un componente, $j$, del gas:

$$
0=(g)_{(\mathrm{i}+1) \mathrm{j}}-(g)_{\mathrm{ij}}+\sum_{\mathrm{k}}^{\mathrm{NR}} v_{\mathrm{k}} \delta_{\mathrm{ik}} \mathrm{R}_{\mathrm{k}}
$$

donde $i=1,2,3,4,5$.

La solución del sistema permite predecir el flujo molar de cada componente y determinar las velocidades globales de reacción (6):

$$
\begin{gathered}
\mathrm{R}_{1}=\frac{(s)_{02}}{3} \\
\mathrm{R}_{3}+\mathrm{R}_{9}=(s)_{03}+\frac{2}{3}(s)_{02} \\
\mathrm{R}_{5}=(s)_{04}+3(s)_{03}+2(s)_{02} \\
\mathrm{R}_{6}=(\mathrm{g})_{64} \\
\mathrm{R}_{7}=(g)_{63} \\
\mathrm{R}_{8}=(g)_{67} \\
\mathrm{R}_{10}=(s)_{06} \\
\mathrm{R}_{11}=(s)_{09}
\end{gathered}
$$

Resulta conveniente expresar el mayor número posible de velocidades de reacción en función de $R_{4}$ y de $R_{5}$ puesto que el comportamiento dinámico del sistema puede sistematizarse en función, principalmente, de estas dos velocidades de reacción. $R_{4}, R_{5}$ y $R_{9}$ pueden expresarse, además, resolviendo las ecuaciones [1-4] en función de las composiciones del gas de tragante. Se puede obtener una expresión completa de las velocidades $R_{1}$ a $R_{11}$ incluyendo las medidas mencionadas de gases de tragante $(g)_{12}=$ $\left(\mathrm{H}_{2}\right)_{1},(g)_{15}=(\mathrm{CO})_{1}$ y $(g)_{16}=\left(\mathrm{CO}_{2}\right)_{1}$ (que se obtienen en continuo del análisis de gases del horno), a partir de las expresiones siguientes:

$$
\begin{gathered}
R_{2}=\gamma R_{4} \\
R_{3}=\frac{R_{5}-(s)_{04}}{3}-R_{9}
\end{gathered}
$$


$\mathrm{R}_{4}=\frac{1}{1+\gamma}\left[(g)_{16}+(g)_{15}-2 \mathbf{R}_{6}-\mathbf{R}_{7}-\mathbf{R}_{10}-(g)_{66}-(g)_{65}\right]$

$$
\begin{gathered}
\mathrm{R}_{5}=\left[(s)_{04}+3(s)_{03}+2(s)_{02}\right] \\
\frac{2(g)_{16}+(g)_{15^{-}}-2(g)_{64}+(m / 2)(g)_{67^{-}}-(g)_{12}-2(g)_{66^{-}}(g)_{65}+(g)_{62}}{2(s)_{06}+3(s)_{02}+4(s)_{03}} \\
\mathrm{R}_{9}=(g)_{62}+(g)_{63}+\frac{m}{2}(g)_{67}-(g)_{12}
\end{gathered}
$$

El examen de los datos experimentales disponibles de la composición del $\mathrm{CO}$ y del $\mathrm{CO}_{2}$ en el interior del horno indica que el punto en el que la concentración de $\mathrm{CO}$ alcanza su máximo, la relación molar $\left(\mathrm{CO} / \mathrm{CO}_{2}\right)=\left((g)_{45} /(g)_{46}\right)$ tiene un valor aproximado de 3. De acuerdo con las suposiciones del modelo, el máximo de esta relación se produce entre las zonas 3 y 4 , por lo que:

$$
\frac{(g)_{45}}{(g)_{46}}=\frac{(g)_{65}+2 \mathbf{R}_{6}+\mathbf{R}_{7}+2 \mathbf{R}_{4}-\mathbf{R}_{5}}{(g)_{66}+\mathbf{R}_{5}-\mathbf{R}_{4}}
$$

$\mathrm{Si}$, de forma más general, se designa el valor del cociente anterior como $E$, se tiene que el valor de $\gamma$, en principio desconocido, es:

$\gamma=\frac{\left[(g)_{16}+(g)_{15}-2 \mathrm{R}_{6}-\mathrm{R}_{7}-\mathrm{R}_{10}\right]}{(1+E) \mathrm{R}_{5}-2 R_{6} \mathrm{R}_{7}}(2+\mathrm{E})-1$

Con todo lo anterior, considerada una alimentación $(s)_{0 \mathrm{j}}$ y $(g)_{6 \mathrm{j}}(j=1,2 \ldots N S$ o $N G)$ al horno y una medida de las variables $(g)_{12},(g)_{15}$ y $(g)_{16}$ con la suposición de que $E$ tome un valor conocido, es posible calcular todas las velocidades de reacción $\mathrm{R}_{\mathrm{k}} \mathrm{y}$ a partir de ellas todas las composiciones o flujos de las corrientes intermedias $(s)_{\mathrm{ij}} \mathrm{y}(g)_{\mathrm{ij}}$. En realidad, la suposición de que $E$ es constante no es muy realista. A partir de los datos expuestos en el capítulo siguiente, se ha considerado que la relación de composiciones queda representada adecuadamente por la correspondiente al equilibrio gas-hierro-wustita en las condiciones de salida del gas de la zona cuarta. De esta forma, $E=E\left(T_{4 \mathrm{~g}}\right)$, de acuerdo con expresiones de equilibrio descritas en la bibliografía (8).

\subsection{Balance de energía}

Se puede plantear un balance de energía al sólido y otro al gas para cada una de las zonas del horno. Se ha supuesto que las temperaturas del sóli- do y del gas son homogéneas en una zona determinada y que, además, tienen el mismo valor que las temperaturas de las corrientes sólida y gaseosa, respectivamente, que salen de ella (9). El número de incógnitas es inicialmente de doce, correspondientes a las temperaturas $T_{\mathrm{is}}$ y $T_{\mathrm{ig}} . T_{0 \mathrm{~s}}$ es la temperatura de la carga de sólidos y $T_{6 \mathrm{~g}}$ la del viento. La fórmula general del balance de energía es la siguiente:

Balance de energía al sólido:

$$
\begin{aligned}
& \sum_{\mathrm{j}}^{\mathrm{NS}}\left[s_{\mathrm{ij}} C_{\mathrm{sij}}\right] \frac{\mathrm{d} T_{\mathrm{is}}}{\mathrm{d} t}=\sum_{\mathrm{j}}^{\mathrm{NS}}\left[(s)_{(\mathrm{i}-1) \mathrm{j}} \bar{c}_{\mathrm{s}(\mathrm{i}-1) \mathrm{j}}\right]\left(T_{(\mathrm{i}-1) \mathrm{s}}-T_{\mathrm{R}}\right)- \\
& \quad-\sum_{\mathrm{j}}^{\mathrm{NS}}\left[(s)_{\mathrm{ij}} \bar{c}_{\mathrm{sij}}\right]\left(T_{\mathrm{is}}-T_{\mathrm{R}}\right)-h_{\mathrm{isg}}\left[T_{\mathrm{is}}-T_{\mathrm{ig}}\right]- \\
& -h_{i p s} A_{\mathrm{i}}\left[T_{\mathrm{is}}-T_{\mathrm{a}}\right]+\sum_{\mathrm{k}}^{\mathrm{NR}}\left[\delta_{\mathrm{ik}}\left(-\Delta H_{\mathrm{k}, \mathrm{i}}\right)\left(1-p_{\mathrm{k}}\right) R_{\mathrm{k}}\right]
\end{aligned}
$$

donde $i=1,2,3,4,5$.

Balance de energía al gas

$$
\begin{aligned}
& \sum_{\mathrm{j}}^{\mathrm{NG}}\left[(g)_{\mathrm{ij}} C_{\mathrm{gij}}\right] \frac{\mathrm{d} T_{\mathrm{ig}}}{\mathrm{d} t}=-\sum_{\mathrm{j}}^{\mathrm{NG}}\left[(g)_{\mathrm{ij}} \bar{c}_{\mathrm{gij}}\right]\left(T_{\mathrm{ig}}-T_{\mathrm{R}}\right)+ \\
& +\sum_{\mathrm{j}}^{\mathrm{NG}}\left[(g)_{(\mathrm{i}+1) \mathrm{j}} \bar{c}_{\mathrm{g}(\mathrm{i}+1) \mathrm{j}}\right]\left(T_{(\mathrm{i}+1) \mathrm{s}}-T_{\mathrm{R}}\right)+h_{\mathrm{isg}}\left(T_{\mathrm{is}}-T_{\mathrm{ig}}\right)- \\
& \quad-h_{\mathrm{ipg}} A_{\mathrm{i}}\left(T_{\mathrm{ig}}-T_{\mathrm{a}}\right)+\sum_{\mathrm{k}}^{\mathrm{NR}}\left[\delta_{\mathrm{ik}}\left(-\Delta H_{\mathrm{k}, \mathrm{i}}\right) p_{\mathrm{k}} R_{k}\right]
\end{aligned}
$$

donde $i=1,2,3,4,5$.

En estado estacionario, los primeros miembros de [20] y [21] son nulos. Las diez ecuaciones de los balances de energía son suficientes para calcular todas las temperaturas $T_{\text {is }}$ y $T_{\text {ig }}$, teniendo en cuenta que se conocen $T_{0 \mathrm{~s}}$ y $T_{6 \mathrm{~g}}$. Para ello, se requiere conocer los valores de los coeficientes de transmisión de calor de gas a sólido $\left(h_{\text {isg }}\right)$, entre el sólido y la pared del horno $\left(h_{\mathrm{ips}} A_{\mathrm{i}}\right)$, entre el gas y la pared $\left(h_{\mathrm{ipg}} A_{\mathrm{i}}\right)$ y la fracción de entalpía comunicada al gas $\left(p_{\mathrm{k}}\right)$. Aunque para ello se pueden seguir diversas estrategias, es ineludible disponer de datos para el horno al que se aplique el modelo.

\section{DATOS EXPERIMENTALES UTILIZADOS EN LA IDENTIFICACIÓN DE PARÁMETROS}

Para la resolución de los balances de materia y energía, es necesario conocer algunos datos fisico- 
químicos del sistema. De acuerdo con las relaciones expuestas en los capítulos anteriores, es preciso determinar la relación molar $\mathrm{CO} / \mathrm{CO}_{2}$ entre las zonas tercera y cuarta para proceder al cálculo de $E$. Además, se precisa estimar el área lateral de cada una de las zonas. Se trata de la mínima información que es imprescindible aportar al modelo a partir de consideraciones a priori. Para ello, se ha empleado información proporcionada por ENSIDESA procedente de la evolución de la composición interna de $\mathrm{CO}$ y $\mathrm{CO}_{2}$ de los gases del interior de un horno alto a lo largo de dos generatrices (paredes NE y SO) en seis niveles de altura. Los datos se tomaron de forma intermitente a lo largo de varios meses.

De los datos disponibles, se puede observar (Fig. 2) que la concentración de $\mathrm{CO}$ disminuye a medida que se asciende en el horno hasta el nivel 18-20 (altura en metros sobre toberas). En el caso del $\mathrm{CO}_{2}$, su concentración en el gas aumenta constantemente hasta estabilizarse en zonas próximas al tragante (Fig. 3). La concentración de CO es distinta según la pared analizada (SO o NE), lo que indica que los gases, a lo largo del horno, no fluyen uniformemente, creándose probablemente canales. De acuerdo con estas observaciones, se puede hacer una estimación inicial de la posición de la zona de reserva térmica, que se debería encontrar aproximadamente entre los niveles 12-18, donde la composición de $\mathrm{CO}$ y $\mathrm{CO}_{2}$ apenas cambia. En un intento de clasificar las zonas del horno, se han propuesto los niveles siguientes:

- Hasta el nivel 6:

Zona de combustión.

- Desde el nivel 6 hasta el 12: Zona cohesiva.

- Desde el nivel 12 hasta el 18:

Zona de reserva térmica.



Fig. 2.- Evolución de la composición de $\mathrm{CO}$ en el gas para distintos niveles del horno.

Fig. 2.- Experimental profile of $\mathrm{CO}$ in rising gas for several heights.

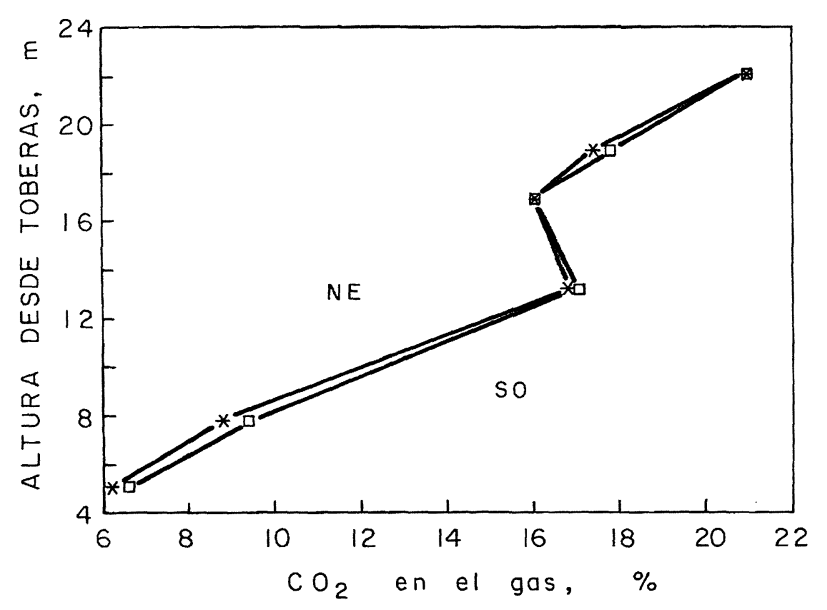

FIG. 3.- Evolución de la composición de $\mathrm{CO}_{2}$ en el gas para distintos niveles del horno.

Fig. 3.- Experimental profile of $\mathrm{CO}_{2}$ in rising gas for several heights.

- Desde el nivel 18:

Zona de reducción de $\mathrm{Fe}_{2} \mathrm{O}_{3}$ y de precalentamiento de la carga.

De acuerdo con los datos tomados, se ha observado que cuando la marcha que tiene lugar en el horno es de tipo central, resulta más difícil determinar la zona de reserva térmica, y ésta parece ser más pequeña que en el caso de marchas periféricas, apareciendo en el primer caso aproximadamente entre los niveles 17-19. A partir de las consideraciones mencionadas, se han estimado unos valores iniciales del área lateral de cada zona.

\section{PREDICCIONES DEL MODELO}

La solución del modelo matemático permite predecir la composición final de todas las corrientes y determinar el perfil interno de temperaturas. En el horno alto es importante efectuar un control preciso de la temperatura debido a su influencia sobre el proceso: el enfriamiento del horno da lugar a escorias muy viscosas y pueden producirse cuelgues de carga o dificultades de colada provocadas por el enfriamiento del crisol. Además, los contenidos de silicio, que condicionan las etapas posteriores del proceso siderúrgico, dependen del estado térmico del horno. El modelo predice el perfil de composiciones en las distintas zonas del mismo: óxidos de hierro ( $\mathrm{FeO}, \mathrm{Fe}_{2} \mathrm{O}_{3}$ y $\mathrm{Fe}_{3} \mathrm{O}_{4}$ ), carbono, composición de $\mathrm{CaO}, \mathrm{Al}_{2} \mathrm{O}_{3}$ y otros óxidos en la escoria (Fig. 4), y fracción de $\mathrm{CO}, \mathrm{CO}_{2}$ y $\mathrm{H}_{2}$ en los gases del horno (Fig. 5). Por otra parte, también permite obtener los valores de temperatura en cada una de las zonas del horno, así como determinar la temperatura del arrabio que cae al crisol (10). 


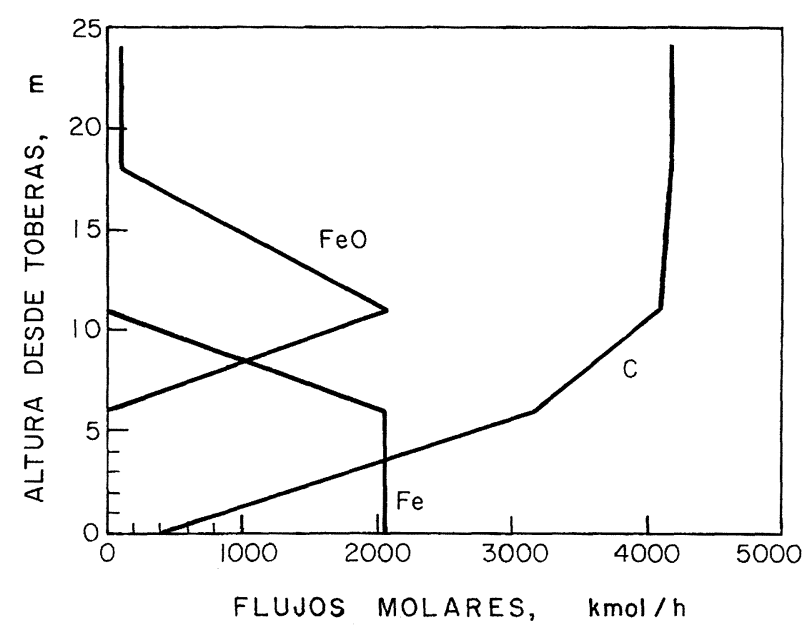

Fig. 4.- Perfiles de composición de carbono, hierro y wustita a lo largo del horno.

Fig. 4.- Calculated profiles of carbon, iron and wustite along the furnace.

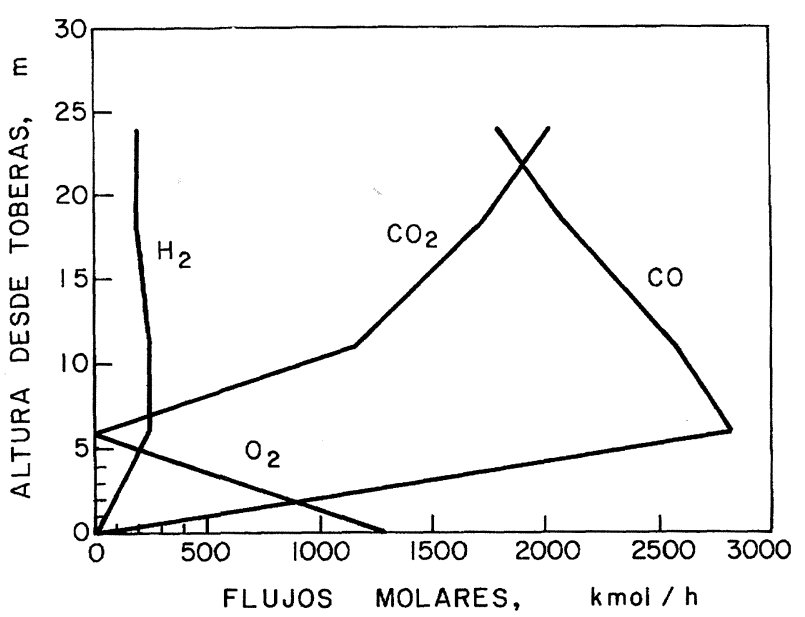

Fig. 5.- Perfiles de composición de $\mathrm{CO}, \mathrm{CO}_{2}, \mathrm{O}_{2}$ $\mathrm{y} \mathrm{H}_{2}$ en el gas.

Fig. 5.-Calculated profiles of $\mathrm{CO}, \mathrm{CO}_{2}, \mathrm{O}_{2}$ and $\mathrm{H}_{2}$ in the gas.

De acuerdo con los datos obtenidos, se ha observado (Fig. 6) que la temperatura del gas es superior a la del sólido en todo el horno excepto en la zona intermedia, de reserva térmica, donde los valores de ambas coinciden. La inversión aparente de temperatura se debe a la forma sencilla que se ha adoptado para el gradiente de temperatura gas-sólido en las ecuaciones [20] y [21]. En la zona inferior tiene lugar la combustión del carbono del coque, elevándose la temperatura hasta aproximadamente 2.000 ${ }^{\circ} \mathrm{C}$. El valor de unos $1.300 \mathrm{~K}$ para el viento a la entrada del horno es el dato experimental y corresponde a las condiciones reales de soplado. Debido a la rápida formación de $\mathrm{CO}$ en las inmediaciones de las toberas, esta temperatura se eleva hasta un valor próximo a la temperatura de llama a una distancia

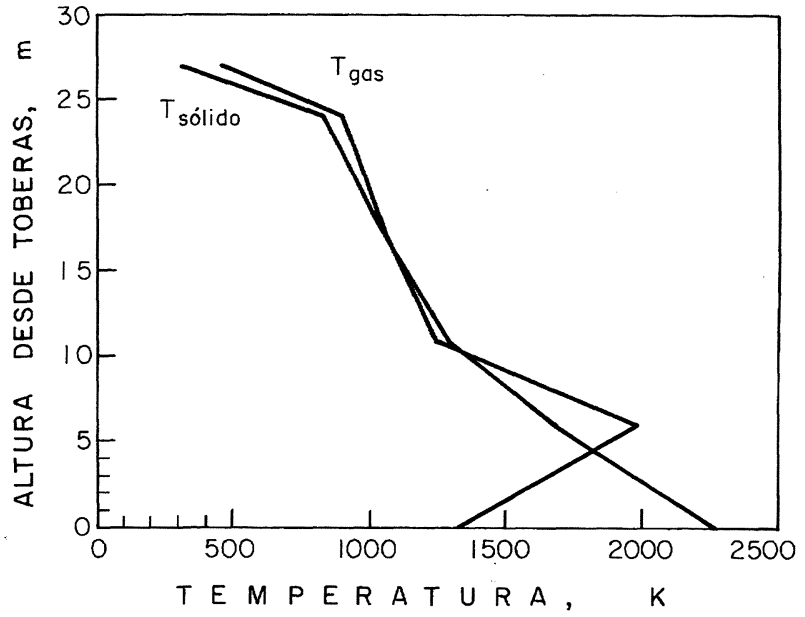

Fig. 6. - Perfil de temperatura según la solución del modelo matemático.

Fig. 6.-Calculated temperatures of solids and gases from the mathematical model.

de poco más de un metro de la misma (8). El modelo que se plantea no trata de representar este fenómeno sino de obtener valores precisos de las temperaturas $T_{4 \mathrm{~g}}, T_{5 \mathrm{~g}}$ y $T_{5 \mathrm{~s}}$ fundamentalmente, que son las claves para el control del proceso. La temperatura de los gases disminuye conforme estos ascienden en el horno, debido a que se produce el calentamiento y la fusión de las cargas y tienen lugar reacciones químicas con un balance global endotérmico. Los perfiles de temperatura obtenidos permiten determinar el estado térmico del horno y la distribución de las zonas. En función de la alteración de las variables que definen la zona de reserva térmica, se puede determinar la altura y características de la zona cohesiva y relacionarla con el tipo de marcha que tiene lugar en el horno. Las pendientes de los perfiles informan de su estado térmico y, por tanto, de qué tipo de cargas se deben añadir, de la temperatura recomendable del viento y de las posibles modificaciones de alguna de las variables en que se basa la acción de control, tales como la humedad del aire y la distribución de la carga. El efecto de cada una de estas variables puede simularse con el modelo matemático $\mathrm{y}$, por tanto, es posible establecer estrategias de comportamiento que permitan predecir las composiciones y temperaturas deseadas para así evitar los comportamientos anómalos del horno.

\section{EFECTO DE ALGUNAS VARIABLES EN LA MARCHA DEL HORNO}

\subsection{Estado estacionario}

En estado estacionario, las derivadas con respecto al tiempo en las ecuaciones [20] y [21] se anulan. 
Sería posible determinar el perfil de temperatura que se produciría en estado estacionario si las condiciones externas de alimentación al horno y la composición y temperatura del gas de tragante fuesen constantes. En concreto, se pueden determinar los estados estacionarios correspondientes a distintas variables de estado que se producen tras una acción de control (automática o manual). A continuación, se presentan ejemplos de respuesta en estado estacionario a las alteraciones de la cantidad de coque (aporte de carbono) en la carga, y la humedad, que son, junto con las modificaciones en el caudal y la temperatura del viento y la introducción de un combustible auxiliar, las principales acciones de control disponibles para dirigir la operación del horno alto.

Se ha considerado un horno que opera en estado estacionario en las condiciones siguientes: $3,26 \mathrm{~kg}$ carbono/kg hematites, $131.000 \mathrm{~m}^{3} \mathrm{~N} / \mathrm{h}$ de aire soplado a $1.330 \mathrm{~K}$ y un contenido de humedad de 38 $\mathrm{g} / \mathrm{m}^{3} \mathrm{~N}$.

En cuanto a la variación de la relación coque/mineral, la figura 7 muestra los perfiles de temperatura de los sólidos en estado estacionario para un horno que opere en las condiciones anteriores y para el caso en el que se efectúen alteraciones de la cantidad de carbono de $\pm 5 \mathrm{~kg}$ carbono/t de hierro en arrabio. El aumento de la cantidad de carbono o de la relación coque/mineral tiene como consecuencia el calentamiento del horno. Recíprocamente, la introducción de menor cantidad de coque lo enfría. El consumo de coque es el principal factor a tener en cuenta en la economía del proceso debido a su coste relativamente elevado. Por ello, se trata de mantener en el nivel más bajo posible sin que se produzcan los efectos mencionados



FIG. 7.- Efecto de la variación de la relación coque/mineral sobre la temperatura del sólido.

Fig. 7.-Effect of a change in the relation cokelore on the temperature of solids. anteriormente relacionados con el enfriamiento excesivo del horno. La alteración provocada por los cambios en la relación coque/mineral afectan considerablemente a todo el horno, aunque con lentitud.

La figura 8 muestra la variación de los perfiles de temperatura del gas en estado estacionario cuando se cambia la cantidad de vapor de agua inyectado con el viento en $\pm 8 \mathrm{~g} / \mathrm{m}^{3} \mathrm{~N}$. Las repercusiones térmicas de este cambio se encuentran limitadas a la zona de combustión y a la cohesiva. La inyección de vapor enfría el horno al aumentar la extensión de la reacción endotérmica entre ésta y el carbono del coque $\left(\mathrm{R}_{7}\right)$.

La variación de la temperatura del aire que se sopla es un factor de gran influencia en la buena marcha térmica del horno. Al aumentar la temperatura del viento, aumenta siempre la producción, por lo que en funcionamiento se está siempre muy cerca del máximo de temperatura compatible con las posibilidades de cada instalación. Por este motivo, su utilización como variable de control es restringida.

\subsection{Consideraciones acerca del comportamiento en estado no estacionario}

La evolución de la temperatura de cada zona en función del tiempo puede obtenerse mediante integración de las ecuaciones diferenciales [20] y [21] siempre que la estimación de los retrasos dinámicos que determinan la inercia térmica de cada zona, dada por $\Sigma\left[\mathrm{s}_{\mathrm{ij}} \mathrm{Cs}_{\mathrm{ij}}\right]$ y $\Sigma\left[\mathrm{g}_{\mathrm{ij}} \mathrm{Cg}_{\mathrm{ij}}\right]$, sea suficientemente precisa. Las principales acciones que pueden tomarse para alterar el estado del horno son la variación de la relación coque/mineral y el cambio de la

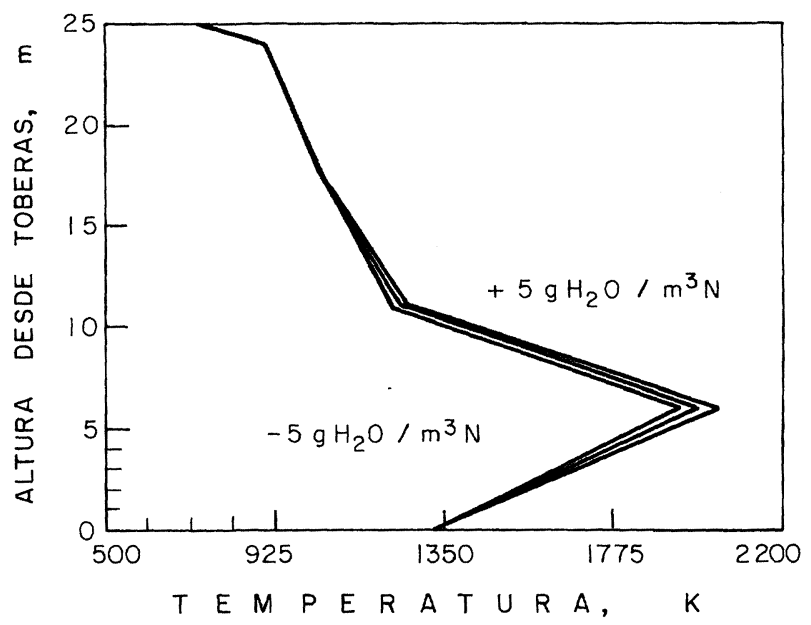

FIG. 8.- Efecto del vapor de agua sobre la temperatura del gas.

Fig. 8.- Effect of steam on the temperature of gases.

Rev. Metal. Madrid, 31 (3), 1995 
humedad y del caudal y la temperatura del aire soplado (viento). Las tres últimas son acciones cuyo efecto se manifiesta a corto plazo. La modificación de la composición del gas reductor es rápida y el equilibrio material se establece a velocidad elevada. Por este motivo, la cantidad de vapor inyectado es la variable más utilizada en el control del horno en situaciones normales (y el motivo por el que siempre se introduce aire húmedo, permitiendo de esta forma elevar rápidamente la temperatura del horno al disminuir la cantidad de vapor que contiene). Cuando varía la relación coque/mineral, la composición de las distintas zonas del horno cambia apreciablemente durante las horas siguientes a la actuación. Sin embargo, esto no invalida la tercera hipótesis del modelo referente al estado estacionario del balance de materia, puesto que las velocidades $R_{k}$ se recalculan para cada instante de tiempo a partir de medidas de las composiciones de los gases de tragante según las ecuaciones [15], [16] y [17]. Esto permite no considerar acumulaciones de materia de forma explícita y, por tanto, separar matemáticamente la resolución de los balances de materia y de energía. Si se considera que tanto los flujos de componentes del sistema como de la temperatura del viento pueden ser función del tiempo, el modelo permite determinar cualquier variable de estado en cualquier instante de tiempo a partir del momento actual y predecir el resultado de una acción de control determinada o de varias superpuestas.

\section{CONCLUSIONES}

El control del horno alto resulta complejo debido a la dificultad de disponer de medidas internas que permitan efectuar un seguimiento del proceso. Por otra parte, las grandes dimensiones del horno dan lugar a que los tiempos de respuesta ante los cambios realizados sean elevados.

Se ha planteado un modelo matemático basado en el comportamiento fisicoquímico del sistema. Se han considerado las reacciones que tienen lugar, las zonas donde estas ocurren y se ha efectuado un análisis de los principales parámetros de comportamiento: coeficientes de transmisión de calor, relación entre los gases que se producen y fracción de entalpía de reacción que se comunica al gas. La solución del modelo permite predecir las composiciones de cada una de las corrientes y el perfil de temperatura dentro del horno. El ajuste de los parámetros de comportamiento se ha efectuado a partir de datos históricos procedentes de hornos en funcionamiento. Se ha realizado un estudio del efecto que la alteración de las variables que constituyen las principales acciones de control tienen sobre el estado estacionario final que se alcanza. Se ha con- siderado el efecto de la relación coque/mineral, de la temperatura inicial del viento y de la cantidad de vapor de agua inyectado. El efecto del vapor de agua es mucho más rápido que el de la variación de la relación coque/mineral, motivo por el cual se emplea para controlar a corto plazo la temperatura en el horno.

\section{Agradecimiento}

Los autores desean expresar su agradecimiento a los técnicos e ingenieros de los hornos altos de ENSIDESA por su interés y apoyo en la realización de este trabajo. El proyecto del que esta comunicación forma parte, se desarrolla dentro del acuerdo CECA 7210-AA/933-934.

\section{Nomenclatura}

$A_{1} \quad$ Área de la pared lateral de la zona $i, \mathrm{~m}^{2}$.

$\bar{c}_{\mathrm{gij}} \quad$ Capacidad calorífica media del componente $j$ del gas entre la temperatura de referencia y $T_{\mathrm{ig}}, \mathrm{kcal} \cdot \mathrm{kmol}^{-1} \cdot \mathrm{K}^{-1}$.

$\bar{c}_{\mathrm{sij}} \quad$ Capacidad calorífica media del componente $j$ del sólido entre la temperatura de referencia y $T_{\text {is }}, \mathrm{kcal} \cdot \mathrm{kmol}^{-1} \cdot \mathrm{K}^{-1}$.

$C_{\text {gij }} \quad$ Capacidad calorífica del componente $j$ del gas a la temperatura de la zona $i\left(T_{\mathrm{ig}}\right)$, $\mathrm{kcal} \cdot \mathrm{kmol}^{-1} \cdot \mathrm{K}^{-1}$.

$C_{\mathrm{sij}} \quad$ Capacidad calorífica del componente $j$ del sólido a la temperatura de la zona $i\left(T_{\mathrm{is}}\right)$, $\mathrm{kcal} \cdot \mathrm{kmol}^{-1} \cdot \mathrm{K}^{-1}$.

E Valor del cociente $(\mathrm{CO})_{4} /\left(\mathrm{CO}_{2}\right)_{4}$.

$g_{\text {ij }} \quad$ Cantidad del componente gaseoso $j$ en la zona $i$, kmol.

$(g)_{\mathrm{ij}} \quad$ Flujo molar de componente gaseoso $j$ que sale de la zona $i, \mathrm{kmol} / \mathrm{h}$.

$h_{\mathrm{i}} \quad$ Altura de cada zona del horno, $\mathrm{m}$.

$\Delta H_{\mathrm{k}, \mathrm{i}}$ Entalpía de la reacción $k$, kcal.

$h_{\mathrm{ipg}}$ Coeficiente de transmisión de calor entre la pared y el gas en la zona $i, \mathrm{kcal} \cdot \mathrm{m}^{2}$. $\mathrm{h}^{-1} \cdot \mathrm{K}^{-1}$.

$h_{\text {ips }} \quad$ Coeficiente de transmisión de calor entre la pared y el sólido en la zona $i, \mathrm{kcal} \cdot \mathrm{m}^{2}$. $\mathrm{h}^{-1} \cdot \mathrm{K}^{-1}$.

$h_{\text {isg }} \quad$ Coeficiente de trasmisión de calor entre sólido y gas en la zona $i, \mathrm{kcal} \cdot \mathrm{h}^{-1} \cdot \mathrm{K}^{-1}$.

$N G \quad$ Número de componentes gaseosos.

$N R \quad$ Número de reacciones.

NS Número de componentes sólidos.

$p_{\mathrm{k}} \quad$ Fracción de la entalpía de la reacción $k$ que se comunica al gas.

$R_{\mathrm{k}} \quad$ Velocidad global de la reacción $k, \mathrm{kmol} / \mathrm{h}$.

$s_{\mathrm{ij}} \quad$ Cantidad de componente sólido $j$ en la zona $i$, kmol. 
$(s)_{\mathrm{ij}} \quad$ Flujo molar de componente sólido $j$ que sale de la zona $i, \mathrm{kmol} / \mathrm{h}$.

$T_{\mathrm{R}} \quad$ Temperatura de referencia, $\mathrm{K}$.

$T_{\mathrm{a}} \quad$ Temperatura del exterior del horno, $\mathrm{K}$.

$T_{\text {ig }} \quad$ Temperatura del gas en la zona $i$ o temperatura de la corriente de gas que abandona la zona $i, \mathrm{~K}$.

$T_{\text {is }} \quad$ Temperatura del sólido en la zona $i$ o temperatura de la corriente sólida que abandona la zona $i, \mathrm{~K}$.

$\gamma \quad$ Relación de velocidades dada por la ecuación [13].

$\nu_{\mathrm{i}} \quad$ Coeficiente estequiométrico.

\section{Subíndices:}

i: $\quad$ Número de zona $(1,2 \ldots 5)$.

j: $\quad$ Número de componente $(1,2 \ldots N S$ o $N G)$.

$\mathrm{k}$ : $\quad$ Número de reacción $(1,2 \ldots N R)$.

s: Sólido.

g: Gas.

\section{Nomenclature}

$A_{1} \quad$ Wall area related to zone $i, \mathrm{~m}^{2}$.

$\bar{c}_{\mathrm{gij}} \quad$ Average specific heat capacity of component $j$ of gas between reference temperature and $T_{\text {ig }}, \mathrm{kcal} \cdot \mathrm{kmol}^{-1} \cdot \mathrm{K}^{-1}$.

$\bar{c}_{\mathrm{sij}} \quad$ Average specific heat capacity of component $j$ of solid between reference temperature and $T_{\text {is }}, \mathrm{kcal} \cdot \mathrm{kmol}^{-1} \cdot \mathrm{K}^{-1}$.

$C_{\text {gij }} \quad$ Specific heat capacity of component $j$ of gas at the temperature of zone $i\left(T_{\mathrm{ig}}\right)$, kcal. $\mathrm{kmol}^{-1} \cdot \mathrm{K}^{-1}$.

$C_{\text {sij }} \quad$ Specific heat capacity of component $j$ of solid at the temperature of zone $i\left(T_{\text {is }}\right)$, $\mathrm{kcal} \cdot \mathrm{kmol}^{-1} \cdot \mathrm{K}^{-1}$.

$E \quad$ Value of $(\mathrm{CO})_{4} /\left(\mathrm{CO}_{2}\right)_{4}$.

$g_{\text {ij }} \quad$ Amount of gas component $j$ in zone $i$, $\mathrm{kmol} / \mathrm{h}$.

$(g)_{\mathrm{ij}} \quad$ Molar flow of gas component $j$ leaving zone $i, \mathrm{kmol} / \mathrm{h}$.

$h_{\mathrm{i}} \quad$ Height of zone $i, \mathrm{~m}$.

$\Delta H_{\mathrm{k}, \mathrm{i}}$ Enthalpy of reaction $k$, kcal.

$h_{\text {ipg }}$ Heat transfer coefficient between wall and gas in zone $i, \mathrm{kcal} \cdot \mathrm{m}^{2} \cdot \mathrm{h}^{-1} \cdot \mathrm{K}^{-1}$.

$h_{\text {ips }} \quad$ Heat transfer coefficient between wall and solid in zone $i, \mathrm{kcal} \cdot \mathrm{m}^{2} \cdot \mathrm{h}^{-1} \cdot \mathrm{K}^{-1}$. $h_{\text {isg }} \quad$ Heat transfer coefficient between gas and solid in zone $i, \mathrm{kcal} \cdot \mathrm{h}^{-1} \cdot \mathrm{K}^{-1}$.

$N G \quad$ Number of gas components.

$N R \quad$ Number of reactions.

NS Number of solid components.

$p_{\mathrm{k}} \quad$ Fraction of the enthalpy of reaction $k$ supplied to gas.

$R_{\mathrm{k}} \quad$ Global reaction rate of reaction $k, \mathrm{kmol} / \mathrm{h}$.

$s_{\mathrm{ij}} \quad$ Amount of solid component $j$ in zone $i$, kmol.

$(s)_{\mathrm{ij}} \quad$ Molar flow of solid component $j$ leaving zone $i, \mathrm{kmol} / \mathrm{h}$.

$T_{\mathrm{R}} \quad$ Reference temperature, $\mathrm{K}$.

$T_{\mathrm{a}} \quad$ Temperature outside furnace wall, $\mathrm{K}$.

$T_{\mathrm{ig}} \quad$ Temperature of gas in zone $i$ or temperature of the gas stream leaving zone $i, \mathrm{~K}$.

$T_{\text {is }} \quad$ Temperature of solids in zone $i$ or temperature of the solid stream leaving zone $i, \mathrm{~K}$.

$\gamma \quad$ Ratio of reaction rates given by equation [13].

$\nu_{\mathrm{i}} \quad$ Stoichiometric coefficient.

\section{Subscripts}

i Subscript denoting zone number $(1,2 \ldots 5)$.

j Subscript denoting component number $(1,2 \ldots$ $N S$ o $N G$ ).

$\mathrm{k} \quad$ Subscript denoting reaction number $(1,2 \ldots$ $N R$ ).

S Solid.

g Gas

\section{REFERENCIAS}

(1) Kilpinen, A. Chem. Eng. Sci., 43 (8), 1988: 1.813-1.818.

(2) CliXBY, G. Ironmaking Steelmaking, 13 (4), 1986: 169-173.

(3) Turkdogan, E.T., Kor, G.J.W. y Fruehan, R.J. Ironmaking Steelmaking, 7 (6), 1980: 268-280.

(4) Lu, W.K., Trans. ISS, 10, 1987: 51-60.

(5) BI, X., TORsSELl, K. y WIJK, O. ISIJ Int., 32 (4), 1992: 481-488.

(6) Hatano, M., Matoba, Y., Otsuka, K., Yoshiki, M. y MIYAGI, T. Stahl Eisen, 101 (4), 1981: 15-21.

(7) Itaya, H., Aratani, F., Kani, A. y Kiyohara, S. Rev. Metall.-CIT, 79 (5), 1982: 443-450.

(8) Voskobóinikov, V.G., KudRIN, V.A. y YÁKushev, A.M. Metalurgia General, Ed. MIR. Moscú, 1982: 83 y 103.

(9) Apraiz-Barreiro, J. Fabricación de Hierro, Aceros y Fundiciones, Vol (I), Ed. Urmo. Bilbao, 1978: 257.

(10) BI, X., Torssell, K. y WiJK, O. ISIJ Int., 32 (4), 1992: 471-480. 